\title{
Searching for Anomalous Longitudinal Profiles with the FRAM Telescope
}

\author{
Jiri Blazek ${ }^{1 \star}$ for the Pierre Auger Collaboration ${ }^{2 \star \star, \star \star \star}$ \\ ${ }^{1}$ Institute of Physics, Czech Academy of Sciences, Prague \\ ${ }^{2}$ Observatorio Pierre Auger, Av. San Martín Norte 304, 5613 Malargüe, Argentina
}

\begin{abstract}
The study of anomalous longitudinal profiles of extensive air showers presents an interesting opportunity to gain additional insight about the character of hadronic interactions at the highest energies. Moreover, the presence of such profiles would represent direct evidence of a light component in the primary cosmic rays of a given energy, independently of hadronic interaction models. The ratio of profiles observed to have a "double bump" structure (with two clearly distinguishable atmospheric depths of maximum particle count) compared to the total number of events can moreover be used to test the predictions of interaction models. However, the majority of such profiles observed at the Pierre Auger Observatory are caused by clouds. Here we describe a method of rapid monitoring of particularly interesting cosmic ray events, using the F/Photometric Rapid Atmospheric Monitor (FRAM) telescope to identify events with clear, cloudless atmospheric background. The function of FRAM is described in detail and the number of triggered events is presented.
\end{abstract}

\section{Introduction}

The study of the ultra-high energy cosmic rays (UHECRs), usually denoting primary particles with energies higher than $10^{18} \mathrm{eV}$, is uniquely positioned to probe hadronic interactions in an energy range well beyond the capabilities of the current generation of colliders. Another interesting and open question is that of the chemical composition of the incoming primary charged particles, see, e.g., [2]. Studies of this type focus on the average features of the longitudinal profile of a shower, such as the mean slant depth of its maximum particle count and the mean variance of this value, as recorded with the nitrogen fluorescence technique. Recent results from the Pierre Auger Observatory [1] indicate a mixed composition which is becoming heavier with energy [3], but the interpretation of the relevant measurements is burdened with large systematic uncertainties related to differences between predictions of various hadronic interaction models. Several other observables sensitive to the chemical composition are being investigated, such as the asymmetry in the risetime of the signal of the surface detectors [4]. But another such observable should in principle be measurable: assuming a light component in the chemical composition, some of the observed longitudinal profiles should feature a distinct second maximum. Such a profile is created by a highly energetic and deeply penetrating particle originating during the first few generations of a shower development. The position of the second maximum and the rate of occurrence of such "anomalous" showers are sensitive both to the chemical composition of the primary flux and

\footnotetext{
^e-mail: blazekj@fzu.cz

$\star \star$ e-mail: auger_spokespersons@fnal.gov

$\star \star \star$ Author list: www.auger.org/archive/authors_2016_09.html
}

to the properties of the hadronic interaction models such as the cross section, elasticity and multiplicity, which are extrapolated from the latest collider data.

The longitudinal profiles are generally very well described by the so called Gaisser-Hillas (G.-H.) function [5], an empirical Ansatz of the form

$$
N_{G H}(X)=N_{\max }\left(\frac{X-X_{0}}{X_{\max }-X_{0}}\right)^{\frac{X_{\max }-X_{0}}{\lambda}} \exp \left(\frac{X_{\max }-X}{\lambda}\right)
$$

where $X$ is the atmospheric slant depth, $N_{G H}$ the number of particles at that given depth, $N_{\max }$ the number of particles at the shower maximum depth $X_{\max }$ and the remaining $X_{0}$ and $\lambda$ are energy-dependent fit parameters without a consistent physical interpretation. The latter two correspond roughly to the depth of a first interaction point and a radiation length, respectively, but the best fit often assigns them unphysical values.

A small fraction $\left(\sim 10^{-3}\right)$ of both the observed and simulated showers features a deeply propagating primary particle, causing the location of a shower maximum to be significantly deeper in the atmosphere than the mean $X_{\max }$ for a given particle type. A similar phenomenon can also occur during the development of a single shower, when a (leading) particle leaves the evolving cascade early during shower development and manages to propagate deeply into the atmosphere. Such a particle then forms a secondary sub-shower, which, if it possesses a significant fraction of the energy of the primary particle, is clearly discernible from the primary sub-shower. The occurrence of such an event is highly unlikely in the case of heavy nuclei acting as primary particles, due to simple kinematic reasons. An Fe-56 nucleus, in the crude approx- 
imation of a superposition model, can be seen as a system of 56 independently interacting nucleons, each possessing approximately $2 \%$ of the total energy. A clearly identified anomalous profile would then present direct evidence for a light component (protons) at a given energy. Clearly, the anomalous profile should be well described by a combination of two regular Gaisser - Hillas profiles $N_{D G H}(X)=N_{G H}^{1}(X)+N_{G H}^{2}(X)$.

\section{Monte Carlo Study}

The rate of occurrence of anomalous showers can be examined by looking at the properties of a statistically large sample of simulated longitudinal profiles. For this kind of study, where the lateral features and the distribution of particles at the ground are of no interest, the CONEX [6] software presents an ideal tool. A range of energy of $10^{17}-10^{20} \mathrm{eV}$ is investigated, divided into seven bins, each with approximately $10^{5}$ simulated events. The EPOS-LHC interaction model is used, the primary particle being a proton incident at a zenith angle of $60^{\circ}$. Three independent methods for the identification of anomalous profiles are devised: one looking at the number of inflexion points present in the profile, the second one making use of existing R00T routines for extracting signal from background and the third routine discriminating the anomalous profiles based on the quality of the fit of the $N_{D G H}(X)$ in comparison to the $N_{G H}(X)$. Results from the first two methods are shown in figures 1 and 2, respectively. Each method depends on one or several parameters that define the doublebump discrimination, three choices of such parameters being shown in each figure. The outcomes of the third method are consistent with the results shown in Figs. 1 and 2. The fraction of anomalous profiles is of the order of $10^{-3}$ and decreases with energy at first, which is expected since the cross section rises with energy [3] and therefore the two maxima become less distinguishable. The origin of the rise in the highest energy bins is not clear and perhaps warrants further study. However this feature is unlikely to be observable with sufficient statistics due to the steeply falling flux of the primary particles at this energy. Qualitatively similar results were previously obtained by C. Baus et al. [7], but with the notable differences of employing pre-LHC era interaction models and smearing the simulated longitudinal profiles to account for the variance present in real fluorescence detectors.

\section{Shoot-the-Shower (StS) Program at the Pierre Auger Observatory}

The largest obstacle in studying the anomalous profiles is the fact that the majority of "bumpy" profiles recorded by real fluorescence detectors are caused by effects of atmospheric origin. There are two main mechanisms that can create an artificial anomalous profile. The first one is the absorption of light between the shower axis and the detector by an obfuscating atmospheric layer - typically a cloud - which consequently can create an apparent minimum in the observed profile shape. The second

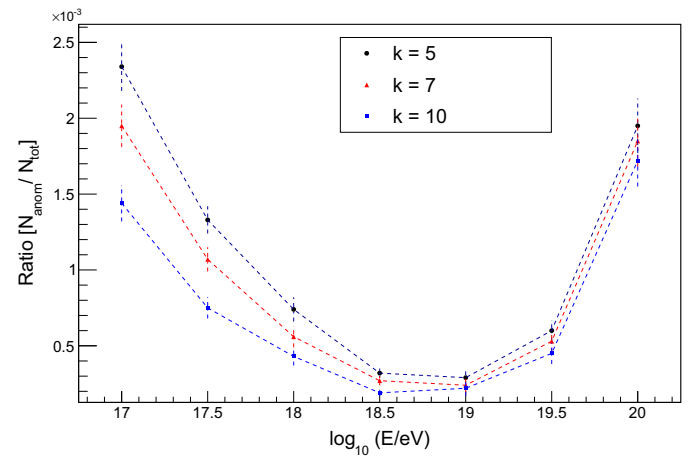

Figure 1. The ratio of identified anomalous profiles $N_{\text {anom }}$ compared to the total number of simulated events $N_{t o t}$, based on the method of identifying the number of inflexion points. The method depends on a parameter $k$, which fixes the minimal separation of two neighboring inflexion points in terms of the number of bins of slant depth. Therefore it also fixes the minimal width of both identified peaks and thus using a higher value of $k$ rejects a set of events corresponding to thinner sub-showers. Three plausible choices of $k$ are shown.

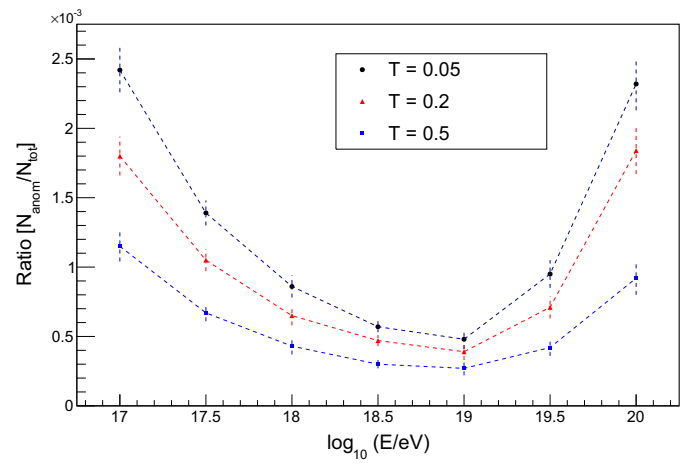

Figure 2. The ratio of identified anomalous profiles based on the method of extracting a number of peaks from background. The method depends on several parameters, the most impactful parameter being the threshold value $T$, which roughly corresponds to the minimum ratio between the amplitudes of the secondary and the primary peak. Three plausible choices of $T$ are shown.

mechanism is the scattering of Cherenkov light when the shower happens to pass through a cloud, creating a second, false peak in the detector due to increased light below the cloud. A proper identification of the presence of such light-affecting layers for each candidate shower is therefore of principal importance. An attempt to address this issue at the Pierre Auger Observatory is the so-called Shoot-the-Shower program. It aims to rapidly record the atmospheric conditions in the vicinity of the axis of a recently recorded shower. It is worth noting that at present there are several types of triggers for the Shoot-the-Shower program, such as particularly-well reconstructed showers, high-energy showers and photon candidates, though the majority of recorded events are still caused by triggers on anomalous profiles. An example of an anomalous event recorded at the Los Leones site of the Pierre Auger Observatory is depicted in Fig. 3. The sharp peak is likely 
caused by the scattering of Cherenkov light on clouds. The FRAM telescope (described below in the text) does not have information about this particular event.

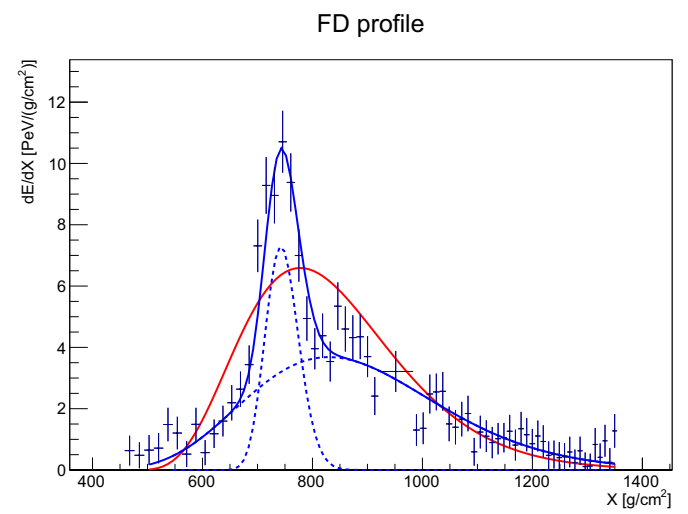

Figure 3. Example of an anomalous fluorescence profile. The red line represents a single G.-H. fit, dashed blue lines represent the fitted sub-showers during a double G.-H. fit and the solid blue line is their combination.

\subsection{The FRAM Telescope}

Several approaches for rapid atmospheric monitoring were tried in the past at the Pierre Auger Observatory. In addition to launching atmospheric balloons after arrival of a very high energy shower to describe actual atmospheric profiles [8], the lidars [9] have been used to obtain the cloud information. The current and running implementation makes use of the so called F/Photometric Rapid Atmosphere Monitor - FRAM [10], located at the Los Leones site. Its main advantage is its completely automatic and autonomous functionality and fully passive operation, which means that it does not interfere with the regular measurements of the fluorescence detectors at all. The working mechanism of FRAM is in principle very simple - it records a series of images in the vicinity of a shower axis, finds stars in these images and compares their apparent brightness with the catalogued values. The FRAM setup consists of several astronomical devices, of which the wide-field camera (field of view $7^{\circ} \times 7^{\circ}$ fixed on a remotely controlled robotic mount) is used for the StS program. When receiving the trigger information, the camera shoots a series of images along the shower axis obtained by an online reconstruction. This whole process takes 5 minutes on average. The images are then copied to a remote storage site and analyzed at a later time.

A first step in the image processing is the application of a dark-field image to correct for the background variance due to any dark current present in the camera system and then the application of a flat-field image to partly correct the optical imperfections of the camera such as vignetting. The resulting calibrated image is then run through an astrometry software suite which identifies potential sources in the recorded field and finds a precise location on the sky. For each identified source, the value of the recorded signal is measured using aperture photometry; the brightest stars are cut from the data-set due to saturation. The remaining sources with apparent magnitudes corrected for the amount of average airmass in the atmosphere along the line of sight are then matched to stars present in the catalog Tycho-2. If then, on a given portion of the image, a region of sources is well-matched to catalogued stars in terms of position but its apparent brightness is significantly lower, it can be inferred that an obfuscating layer was present in that part of the image.

This whole process can be made visually much clearer when plotting all the images from a given shower scan into one picture, thus covering a wide range of airmasses. A theoretical fit of the expected extinction can be made and sets of stars differing significantly and uniformly from the curve of the fit mark regions with light-affecting layers present. Examples of such plots can be seen in Figs. 4 and 5. In the first figure, there are three visible regions with significant variations from the expected fit, at around 7, 13 and 20 degrees of altitude. The latter two regions represent clouds with an average optical thickness of around 0.3 (denoted as $\tau$ and defined as $N_{\text {apparent }} / N_{\text {expected }}=e^{-\tau}$ ), which in the linear approximation thus represents roughly $30 \%$ attenuation in terms of the absolute photon flux. The presented method is sensitive to minimal optical depths of approximately 0.2 . The presence of such regions of significant attenuation would cause the corresponding shower to be discarded for the purposes of the double-bump analysis. Fig. 5 shows a significantly cloudier profile, but note that the stars, though strongly attenuated, are still visible. In many of the recorded images there are wide regions with no discernible stars and then the analysis procedure fails during the first step of astrometry. Such profiles are typical for nights with heavy cloud coverage.

For purposes of the double-bump analysis, the profiles are inspected visually by a member of the FRAM team and only the events with no obvious deviations from the extinction fit are selected and tagged as having a clear atmospheric background. A series of cuts is then applied to the reconstructed fluorescence profiles in order to try to identify the actual anomalous events amid the much larger set of events caused simply by the intrinsic random variations in the corresponding fluorescence profiles. The set of cuts includes the requirement of a significant improvement of the double G.-H. fit over a single G.-H. fit, both maxima being present in the field of view, a minimal track length between the two fitted maxima and other cuts.

\subsection{Number of StS Events Recorded by FRAM}

The FRAM telescope was originally installed in 2005 and has undergone many hardware improvements. The current wide-field camera, producing best results in the StS program, was installed in March 2013. In this analysis the dataset starting from March 2013 is presented. The dataset extends up to April 2016, with newer data still waiting to be analyzed. During this roughly three-year period, the FRAM telescope registered 1788 events with at least 4 pictures per scan recorded ("complete events"). Of these, 659 were identified as showers with a clear atmospheric background. Of this number, 399 events were shot after receiving a double-bump trigger. These numbers 


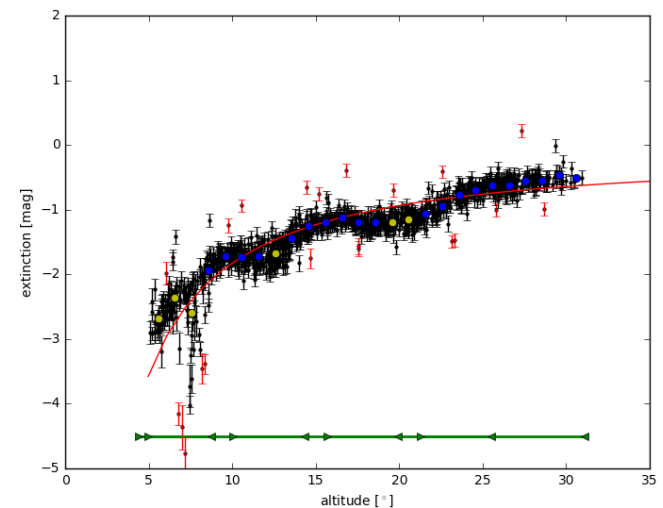

Figure 4. Example of an extinction plot for a FRAM event. The quantity shown on the $y$-axis is the extinction in magnitudes, corresponding to the combination of aerosol and molecular scattering. On the $x$ axis is the altitude, i.e. the angle between the horizon and the line of sight. Each black dot represents an identified light source. The red line is a fit of total extinction due to molecular and aerosol scattering. An ideal cloudless event would have all points clustered along this line, as the attenuation in such a case depends only on the amount of air in the direction of the star. The airmass, and hence also the attenuation, increases towards the horizon. The large dots represent average values, their color indicating their distance to the extinction fit, where blue stands for values within one standard deviation and yellow for values outside of this range. The green lines at the bottom represent the boundaries of each of the shots taken by the camera. The shots have significant overlap in altitude.

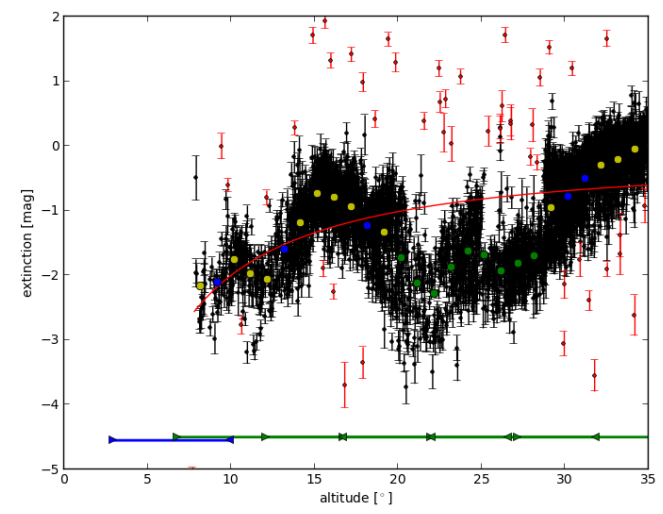

Figure 5. Example of an extinction plot for a cloudy event.

are summarized in Table 1. To compare the resulting rate of anomalous events to the Monte Carlo predictions, the trigger efficiency together with the duty cycle of the entire $\mathrm{StS}$ system need to be properly estimated. This procedure

Table 1. Number of StS events recorded by FRAM between March 2013 and April 2016.

\begin{tabular}{c|c|c}
\hline complete events & no clouds & double bump trigger \\
\hline 1788 & 659 & 399 \\
\hline
\end{tabular}

is not straightforward and is the subject of ongoing work within the Pierre Auger Collaboration.

\section{Conclusions}

Finding a number of clearly anomalous events in a given energy bin could test the parameters of hadronic interaction models which are extrapolated from collider energies. The ratio of expected double-bump events depends both on the elasticity of the leading interactions and on the cross section of the deeply propagating particle. Some predictions using a current hadronic interaction model are presented in section 2. The number of false positives present in the dataset of the Pierre Auger Observatory dominates the expected number of actual anomalous events. A very efficient rejection of events affected by clouds is thus needed. A method making use of the FRAM telescope is described and the resulting number of candidate events is presented in section 3.2. Analysis of this dataset is ongoing. For example, another kind of false positive event may be created by fluctuations present in the electronics of the fluorescence detectors. This needs to be properly accounted for using a Monte Carlo approach. Moreover, the efficiency of the whole StS chain needs to be correctly estimated. Only then can the desired doublebump ratio be obtained.

The work is supported by the Grant Agency of the Czech Republic 14-75101S and by projects MSMT LG15014 and LM2015038.

\section{References}

[1] A. Aab et al. [Pierre Auger Collaboration], Nucl. Instrum. Meth. A 798, 172 (2015), [arXiv:1502.01323 [astro-ph.IM]].

[2] K. H. Kampert and M. Unger, Astropart. Phys. 35, 660 (2012).

[3] A. Aab et al. [Pierre Auger Collaboration], Contributions to the 34th International Cosmic Ray Conference, (ICRC 2015) [arXiv:1509.03732 [astro-ph.HE]].

[4] A. Aab et al. [Pierre Auger Collaboration], Phys. Rev. D 93, no. 7, 072006 (2016) [arXiv:1604.00978 [astro-ph.HE]].

[5] Proc. 15th ICRC 1977, Plovdiv, 8 353, 1977.

[6] T. Bergmann et al., Astropart. Phys. 26, 420 (2007), [astro$\mathrm{ph} / 0606564]$.

[7] C. Baus, R. Engel, T. Pierog, R. Ulrich and M. Unger, Proc. 32nd ICRC 2011, [arXiv:1111.0504 [astro-ph.HE]].

[8] B. Keilhauer [Pierre Auger Collaboration], Astrophys. Space Sci. Trans. 6, 27 (2010).

[9] J. Abraham et al. [Pierre Auger Collaboration]: Astropart. Phys. 33, 108 (2010) [arXiv:1002.0366 [astro-ph.IM]].

[10] J. Ebr et al., Proceedings of the 3rd Astrorob workshop 45, 53 (2014). 